\title{
Otimização do Despacho Hidrotérmico utilizando Lagrangeano Aumentado e SPG
}

\author{
Ana P. Oening, Débora C. Marcilio, Marcelo R. Bessa \\ Divisão de Sistemas Elétricos - DVSE, LACTEC \\ 81531-980, Centro Politécnico da UFPR, Curitiba, PR \\ E-mail: debora@lactec.org.br \\ Luiz C. Matioli \\ Universidade Federal do Paraná - Departamento de Matemática \\ 81531-990, Centro Politécnico, Curitiba, PR \\ E-mail: matioli@ufpr.br
}

\begin{abstract}
Resumo: Neste trabalho é apresentada a utilização do método Lagrangeano Aumentado, em conjunto com o método Gradiente Espectral Projetado (SPG), para resolução do problema de otimização do despacho hidrotérmico do Sistema Interligado Nacional (SIN). Foi utilizado um sistema bem representativo do SIN, com 111 usinas hidráulicas e 32 usinas térmicas, distribuídas em cinco subsistemas, para validar e testar a metodologia adotada. Os resultados obtidos se mostraram bem satisfatórios, tanto sob a ótica do problema do despacho quanto do desempenho computacional do método.
\end{abstract}

\section{Introdução}

Atualmente, o Operador Nacional do Sistema Elétrico (ONS) utiliza uma cadeia de softwares desenvolvidos pelo Centro de Pesquisas de Energia Elétrica (CEPEL), onde o modelo principal é o NEWAVE que trata da otimização do despacho hidrotérmico no médio prazo. $\mathrm{O}$ NEWAVE foi desenvolvido com base na tecnologia de Programação Dinâmica Dual Estocástica (PDDE) [12] e [13], cuja base consiste na decomposição de Benders [1] e na hipótese simplificadora de reservatórios equivalentes. No entanto deve-se ressaltar que desde a criação da primeira versão desses softwares, o Setor Elétrico Brasileiro (SEB) sofreu grandes transformações: o SEB era predominantemente estatal e centralizado, com forte predominância hidrelétrica e com uma folga na geração que permitia acomodar as imprecisões decorrentes das linearizações adotadas.

Dessa forma, considera-se de grande relevância para o SEB a pesquisa que envolve o desenvolvimento de outros modelos de otimização do despacho hidrotérmico, que melhor se adeque ao cenário atual, visando atender ao mercado de energia elétrica com o menor custo possível e garantindo a oferta futura de recursos energéticos.

O problema do despacho hidrotérmico consiste na otimização de quais usinas devem operar e quais devem ficar de reserva, de modo a preservar o volume dos reservatórios para serem utilizados quando necessário, ou seja, a principal decisão é quanto se deve despachar em cada usina hidrelétrica. Se a decisão tomada for utilizar a água no presente, (despachando as usinas hidrelétricas) e no futuro as afluências forem baixas, ter-se-á um custoso déficit de energia. Por outro lado se a decisão for de economizar água (despachar as usinas térmicas) e as afluências futuras forem altas, o resultado será em vertimento de água, o que significa que não foi aproveitado todo o potencial hidráulico do sistema.

A proposta deste trabalho tem como objetivo otimizar o problema do despacho hidrotérmico formulado como um problema de otimização não linear, tendo como objetivo a minimização dos custos operacionais da geração térmica e do custo de eventuais déficits de energia. As restrições consideradas são o atendimento a demanda, balanço hídrico e defluência mínima total para o reservatório. Adicionalmente, são consideradas as restrições operativas que estão representadas pelos limites inferior e superior das variáveis.

A modelagem do despacho hidrotérmico recai em um problema de otimização não linear de grande porte, não convexo e diferenciável, cuja solução depende de métodos de otimização robustos. Este problema tem sido alvo constante de pesquisa e versões iniciais do 
desenvolvimento proposto foram mostradas nos artigos [8] e [10], onde foi apresentada a modelagem do problema do despacho hidrotérmico, o uso do Método de Pontos Interiores aplicado ao problema do despacho hidrotérmico [8] e uma comparação entre os métodos Lagrangeano Aumentado e Pontos Interiores [10]. O objetivo do presente trabalho é apresentar a utilização do método Lagrangeano Aumentado juntamente com o SPG - Gradiente Espectral Projetado, que são métodos conhecidos na literatura por resolver problemas com as características do problema do despacho hidrotérmico. A metodologia adotada para a utilização do método Lagrangeano Aumentado se baseia nos artigos [2], [3] e [4].

\section{Problema do Despacho Hidrotérmico}

O modelo considerado na otimização do despacho hidrotérmico consiste na minimização do valor presente dos custos de geração térmica e de déficit. $\mathrm{O}$ custo de geração térmica é uma função que representa o custo de cada usina térmica para cada período e é aproximada por um polinômio de grau dois. O custo de déficit é uma função que representa o custo de déficit do subsistema, ou seja, representa o impacto causado pelo não suprimento da demanda de energia e é aproximado por um polinômio de segundo grau.

As restrições levam em consideração as restrições operativas das usinas, balanço hídrico, atendimento a demanda, defluência mínima total dos reservatórios e limites das variáveis.

Vale ressaltar que a modelagem do despacho hidrotérmico utilizada nessa pesquisa leva em consideração tanto as usinas hidrelétricas quando as usinas termelétricas de forma individualizada, agregando apenas as usinas pertencentes a um mesmo subsistema na restrição de atendimento a demanda. Uma maior discussão sobre a modelagem do despacho hidrotérmico pode ser encontrada em [8] e [10].

O problema do despacho hidrotérmico, com as características abordadas, quando escrito matematicamente tem o seguinte formato:

$\begin{array}{cc}\text { minimizar } & f(x) \\ \text { sujeito à } & g(x)=0 \\ & A x=b \\ & C x \leq c \\ & l \leq x \leq u\end{array}$

onde $f: \mathbb{R}^{\mathrm{n}} \rightarrow \mathbb{R}$ é a função objetivo não linear, g: $\mathbb{R}^{\mathrm{n}} \rightarrow \mathbb{R}^{\mathrm{m}}$ são restrições não lineares que representam o atendimento à demanda e a geração fixada da usina, $\mathrm{Ax}=\mathrm{b}$ e $\mathrm{Cx} \leq \mathrm{c}$ são restrições lineares que representam, respectivamente, o balanço hídrico e defluência total, $\mathrm{A}, \mathrm{C} \in \mathbb{R}^{\mathrm{p} \times \mathrm{n}}, \mathrm{b}, \mathrm{c} \in \mathbb{R}^{\mathrm{p} \times 1}$, e $\mathrm{l} \leq \mathrm{x} \leq \mathrm{u}$ representam os limites inferior e superior das variáveis (também chamadas de restrições de canalização ou caixa), $\mathrm{x}, \mathrm{l}, \mathrm{u} \in \mathbb{R}^{\mathrm{n}}$. $\mathrm{O}$ vetor $\mathrm{x}$ é a variável de decisão, que no caso do problema de despacho hidrotérmico envolve: geração térmica, vazões vertida e turbinada, volume do reservatório, intercâmbio de energia entre subsistemas e déficit.

\section{Lagrangeano Aumentado}

Métodos de Lagrangeanos Aumentados [11] são iterativos e baseados em penalização e a cada iteração transformam o problema original restrito em um problema irrestrito. Para tanto a estratégia utilizada é a de penalização. As restrições consideradas de difícil trato são acrescidas a função objetivo juntamente com um parâmetro de penalidade e multiplicadores de Lagrange.

Assim, o método é iterativo e cada iteração resolve um problema irrestrito, gerado pela penalização das restrições. $O$ critério de convergência utilizado nesse processo é a condição de otimalidade e viabilidade com relação ao problema original, onde a cada passo em que esses critérios não forem satisfeitos os multiplicadores de Lagrange e o parâmetro de penalidade são atualizados. 
O método Lagrangeano Aumentado implementado emprega a função penalidade definida por Powell Hestenes e Rockafellar [16], as atualizações dos multiplicadores de Lagrange são realizadas de maneira usual forçando satisfazer as condições de KKT. A atualização do parâmetro de penalidade se baseia na metodologia adotada no artigo [2], onde os autores monitoram o atendimento as restrições, para aumentar, diminuir ou manter constante esse parâmetro.

A metodologia adotada para a utilização do método Lagrangeano Aumentado se baseia nos artigos [3] e [4], onde os autores penalizam as restrições lineares de desigualdade e igualdade e não linear de desigualdade. Dessa a penalização do problema (1) resulta em um problema de minimização onde a função objetivo é a função Lagrangeano Aumentada restrita a limitações nas variáveis.

$$
\begin{array}{cc}
\text { minimizar } & L\left(x, \lambda^{k}, \mu^{k}, \rho^{k}\right) \\
\text { sujeito à } & l \leq x \leq u
\end{array}
$$

onde $\lambda^{k}$ e $\mu^{k}$ são os multiplicados de lagrange das restrições de igualdade e desigualdade respectivamente e $\rho^{k}$ é o parâmetro de penalidade. A cada iteração é realizado uma minimização na variável $x \operatorname{com} \lambda^{k}, \mu^{k}$ e $\rho^{k}$ fixos na iteração $k$.

Essa classe de problemas restritos (2) pode ser resolvida pelo método do Gradiente Espectral Projetado, descrito na sequência.

\section{Gradiente Espectral Projetado}

O método SPG se origina do principio que o método do Gradiente Projetado é de fácil implementação e bastante eficiente. A partir desse conceito, os autores em [3] combinaram esse método a dois ingredientes de otimização. Primeiro, estenderam as estratégias de globalização típicas associadas a estes métodos para o esquema de busca linear não monótona desenvolvido por Grippo, Lampariello e Lucidi em [7] para o método de Newton, o que representou uma grande melhoria em relação aos métodos de gradiente projetado tradicionais. Segundo, propuseram a associação do passo espectral, introduzido por Barzilai e Borwein [5] e analisado por Raydan [15]. O passo espectral é um quociente de Rayleigh relacionado com uma média da matriz Hessiana. Esta escolha do tamanho do passo requer pouco esforço computacional e aumenta a velocidade de convergência dos métodos de gradiente projetados.

\section{Sistema Teste e Resultados}

A metodologia atual adota a hipótese simplificadora de agregação dos reservatórios que operam em uma mesma região, no entanto, estas simplificações podem omitir características importantes do sistema, tornando-o pouco flexível e colocando em risco a confiabilidade dos resultados. Para os testes apresentados nesse trabalho foram utilizados séries sintéticas hidrológicas geradas a partir da metodologia apresentada no artigo [6].

O sistema teste considerado utilizado é composto por 111 usinas hidráulicas e 32 usinas térmicas, distribuídas em cinco subsistemas. Desses subsistemas, quatro seguem as características dos subsistemas Sul, Sudeste, Nordeste e Norte e o quinto é formado somente pela usina Itaipu, com a consideração de possuir somente geração e não possuir demanda.

O problema formulado com essas características tem 22.560 variáveis e 13.620 restrições, sendo que as restrições de caixa não foram consideradas, pois elas não foram penalizadas e o número de variáveis representa apenas as variáveis relacionadas ao problema, não sendo considerados na contagem os multiplicadores de Lagrange.

Foram consideradas 200 séries sintéticas representando uma gama de situações hidrológicas [6], dessa maneira são otimizados 200 problemas do despacho hidrotérmico com a finalidade de validar a metodologia proposta. 
Todas as rotinas foram programadas em MATLAB 2010a. Para agilizar o processamento das 200 séries sintéticas foi utilizado um cluster composto por um head-node (nó de controle) e 18 worker-nodes (nós de processamento). Cada um dos nós do cluster é composto por dois processadores com seis núcleos de processamento físico e mais seis núcleos de processamento lógico o que totaliza um poder de processamento de 24 núcleos por nó, totalizando 432 núcleos de processamento. A distribuição das séries nos núcleos de processamento foi feito por meio de um $J o b$, o qual foi responsável por alocar seis núcleos de processamento para cada uma das séries, sendo que para cada conjunto o código foi o mesmo, variando apenas os dados de entrada.

Dos 200 problemas otimizados o Lagrangeano Aumentado e o SPG otimizaram 128 problemas, quando analisada a condição hidrológica do restante dos problemas constata-se que não eram favoráveis, ou seja, as afluências eram baixas e a geração das térmicas não era suficiente para suportar os períodos de seca. Para ilustrar esse fato, foram selecionados dois problemas, o primeiro foi otimizado utilizando os dados da série sintética 4, onde ocorreu a convergência (Figura 1) e o segundo foi otimizado utilizando os dados da série sintética 186, que não convergiu (Figura 2).

Nas Figura 1 e Figura 2 estão representadas a soma por mês da geração térmica (GT), da geração hidráulica $(\mathrm{GH})$ e da afluência, dessa pode-se analisar o comportamento das gerações em afluências altas e baixas.

A série sintética 186 possui períodos de pouca afluência, com isso quando o problema é otimizado a demanda não é atendida, pois as usinas térmicas consideradas no sistema não possuem potência suficiente para complementar a geração das usinas hidráulicas.

Porém analisando o despacho do problema utilizando a série sintética 4 , o qual tem períodos de afluências altas, pode-se notar que a geração térmica atua como complemento da geração hidráulica nos períodos onde a afluência diminui.

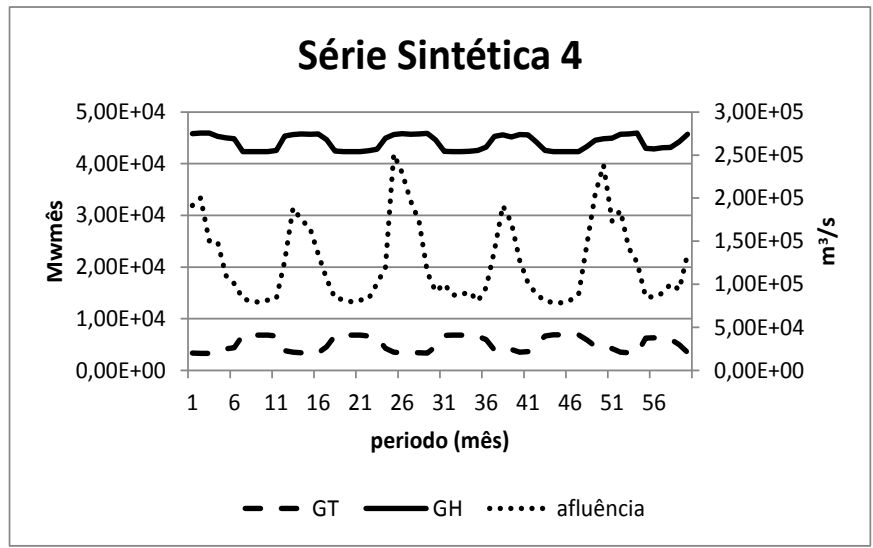

Figura 1: Despacho hidrotérmico da série sintética 4

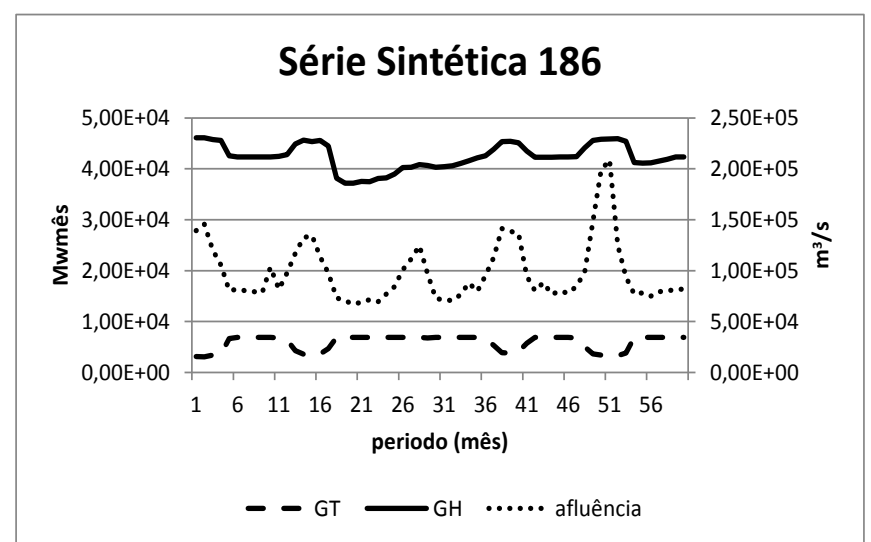

Figura 2: Despacho hidrotérmico da série sintética 186 


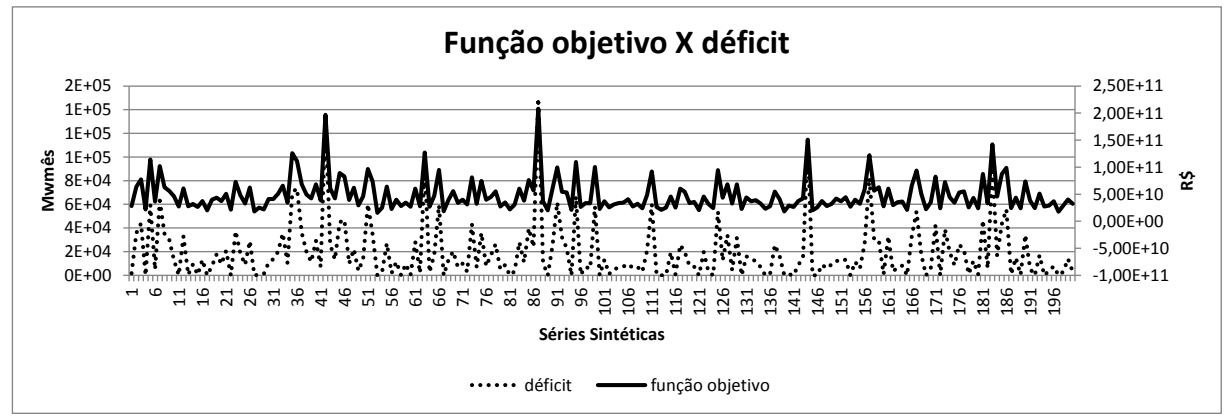

Figura 3: Função objetivo versus déficit

A Figura 3 contém a soma da função objetivo e do déficit por série sintética. Analisando esse gráfico tem-se que quanto maior o déficit, maior o valor da função objetivo, confirmando a adequação da modelagem.

A média do tempo de processamento para cada problema foi de 5 horas, porém com o uso do cluster na distribuição das tarefas, o tempo total de processamento foi de 18 horas, o que viabiliza os estudos realizados.

Comparando os resultados apresentados aqui com os discutidos em [8] e [10] tem-se que os métodos Lagrangeano Aumentado e o SPG mostraram-se mais robustos, pois otimizaram problemas com dimensões maiores e com uma variedade maior de séries hidrológicas.

\section{Conclusão}

O método Lagrangeano Aumentado e o método Gradiente Espectral Projetado mostraram-se, durante esta pesquisa, mais constantes, com resultados homogêneos para as diferentes séries sintéticas. Para os casos onde as séries não eram favoráveis, proporcionando aos métodos uma dificuldade maior para alocar as usinas térmicas de modo a minimizar a sua geração, atender a demanda e satisfazer restrições como os limites de intercâmbio e as próprias limitações de geração da usina, a metodologia proposta também se mostrou bastante confiável.

\section{Referências}

[1] Benders J. F., "Partitioning Procedures for Solving Mixed-Variables Programming Problems", Numerische Mathematik, vol. 4, 1962, pp. 238-252.

[2] Birgin E. G., Martínez J. M., "Augmented Lagrangian Method with Nonmonotone Penalty Parameters for Constrained Optimization", Computational Optimization and Applications, 2011.

[3] Birgin E. G., Martínez J. M., and Raydan M., "Nonmonotone Spectral Gradient Methods on Convex Sets", SIAM Journal on Optimization, vol. 10, 2000, pp. 1196-1211.

[4] Birgin E. G., Martínez J. M., and Raydan M, "SPG: Software for Convex Optimization", ACM Transactions on Mathematical Software, vol. 27, 2001, pp. 340-349.

[5] Borwein J. M., Barzilai J., "Two Point Step Size Gradient Methods", IMA Journal of Numerical Analysis, vol. 08, 1988, pp. 141-148.

[6] Detzel, D. H. M.; Bessa, M. R.; Mine, M. R. M. Amostragem de Séries Sintéticas Hidrológicas. Revista Brasileira de Recursos Hídricos. Aceito para publicação no Vol. 18, $\mathrm{n}^{\mathrm{o}}$ 4, out./dez. 2013.

[7] Grippo L., Lampariello F. and Lucidi S., " A Nonmonotone Line Search Technique for Newton's Method", SIAM Journal on Numercial Analysis, vol. 23, 1986, pp. 707-716.

[8] Kleina M., Marcilio D.C, Oening A.P., Bessa, Matioli L.C, O Método de Pontos Interiores Aplicado ao Problema do Despacho Hidrotérmico. CNMAC2012, pp 81-87-1168, 2012 
[9] Luenberger D. G., Linear and Nonlinear Programming, Springer, Nova York, 2005.

[10] Marcilio D.C, Oening A.P., Bessa, M.R., Matioli L.C., Kleina M., M. Bloot, Pontos Interiores versus Lagrangeano Aumentado na Otimização do Despacho Hidrotérmico. CNMAC2012, pp 1162-1168, 2012.

[11] Nocedal J., Wright S., Numerical Optimization. Springer, Nova York, 2005.

[12] Pereira M. V. F., "Optimal Stochastic Operations Scheduling of Large Hydroelectric Systems", International Journal of Electrical Power \& Energy Systems, vol. 11, 1989, pp. 161-169.

[13] Pereira M. V. F. and Pinto L. M. V. G., "Stochastic optimization of a Multireservoir Hydroelectric Systems: a Decomposition Scheduling”, Water Resources Research, vol. 21, 1985, pp. 779-792.

[14] Quintana V. H., Torres G. L., "On a nonlinear multiple-centrality-corrections interiorpoint method for optimal power flow", IEEE Transactions on Power Systems, vol.16, no. 2, 2001, pp. 222-228.

[15] Raydan M. "On the Barzilai and Borwein choice of Steplenght for the Gradient Method”, IMA Journal of Numerical Analysis, vol. 13, 1993, pp. 321-326.

[16] Rockafellar R. T, A dual approach for solving nonlinear programming problems by unconstrained optimization, Mathematical Programming, vol. 5, 1973, pp. 354-373. 